\title{
Huge coherent acoustic phonon oscillation induced by piezoelectric field in InGaN/GaN multiple-quantum-wells
}

\author{
Chi-Kuang Sun ${ }^{\mathrm{a}}$, Jian-Chin Liang Lianga, Amber Abare ${ }^{\mathrm{b}}$, Larry Coldren ${ }^{\mathrm{b}}$, and \\ Steven P. DenBaars ${ }^{b}$
}

a) Institute of Electro-Optical Engineering and Department of Electrical Engineering, National Taiwan University, Taipei 10617, TAIWAN R.O.C. E-mail: sun@cc.ee.ntu.edu.tw. b) Department of Electrical and Computer Engineering, University of California, Santa Barbara,
CA 93106, USA.

\section{Summary}

Progress in ultrafast spectroscopy technology has enabled us to generate and to directly observe the coherent oscillation of phonon modes. In semiconductors, carriers can be excited by ultrashort laser pulses in a spatial area much larger than one unit cell and the excited carrier populations (with wave vector $\mathrm{q} \cong 0$ ) are coupled to the corresponding optical phonon modes. Some of these optical phonon modes can behave like classical oscillators and have non-zero timedependent displacements. The corresponding modulation of the material dielectric constant could then be observed by changes in the intensities of transmitted or reflected probe light pulses $[1,2]$.

In bulk semiconductors, no specific acoustic phonon mode can be selectively excited by $\mathrm{q} \cong 0$ carriers, and no coherent acoustic phonon oscillation has been observed. Semiconductor superlattices exhibit zone-folding of the acoustic branches within the mini-Brillouin zone due to the artificial periodicity, enabling the observation of coherent oscillation of the zone-folded acoustic phonons in AlAs/GaAs superlattices [3,4]. However, exact integer number of atomic monolayers and high periodicity $(>60)$ were required and the observed reflection modulation $\Delta R / R$ was extremely small, on the order of $10^{-5}$.

In this presentation, we will demonstrate for the first time that with periodic distribution of photoexcited carriers with $q=2 \pi / a$, where a is the period width, carriers can be coupled to the selective acoustic phonon mode with the corresponding $q$. With strain-induced piezoelectric-field, strong coherent acoustic-phonon oscillation can thus be easily initiated and the corresponding huge absorption modulation can be induced. We can easily observe transmission modulation $\Delta \mathrm{T} / \mathrm{T}$ on the order of $10^{-2}$. Unlike its optical phonon counter-part, the frequency of coherent acoustic phonon oscillation can be widely tuned by changing the sample period width.

The experiments were performed at room temperature on 14 periods InGaN/GaN multiplequantum-wells (MQWs). Because of the large piezoelectric constants along the [0001] orientation in group III nitrides, high strain-induced piezoelectric field is expected (on the order of $\mathrm{MV} / \mathrm{cm}$ ). Femtosecond transmission pump-probe measurements were performed using a modelocked Ti:sapphire laser. The laser output pulses were frequency-doubled using a BBO crystal to reach the bandgap energy of InGaN/GaN MQWs. The frequency doubled pulses had a pulsewidth of $150 \mathrm{fs}$ at a wavelength of $380 \mathrm{~nm}$ measured by performing autocorrelation in a bulk GaN sample. The femtosecond pump pulses photo-excited carriers in the MQWs. Due to the periodic distribution of the photoexcited carrier population, carriers were easily coupled to the selective acoustic phonon mode with $\mathrm{q}=2 \pi / \mathrm{a}$, where $\mathrm{a}$ is the MQW period width, during the relaxation processes of electrons and holes. Coherent oscillation was impulsively induced by carrier screening of the piezoelectric field in QW by photoexcited carriers. The induced coherent oscillation with non-zero time-dependent displacement would thus modulate the strain-induced piezoelectric field and result in transmission change through quantum confined Stark effect (QCSE). A cosine-like oscillation should thus be observed. Figure 1 shows the measured probe transmission changes as a function of probe delay for different MQWs with excitation photon 
energies above the absorption bandgap. The normalized transmission changes $\Delta \mathrm{T} / \mathrm{T}$ are on the order of $10^{-1}$. After photoexcitation of carriers that caused transmission increases at zero timedelay, a clear cosine-like transmission oscillation is observed with $\Delta \mathrm{T} / \mathrm{T}$ on the order of $10^{-2}$. We attribute this huge oscillation to coherent longitudinal-acoustic (LA) phonon oscillation in the QW growth direction (c-axis). The oscillation frequencies varied from $1.23 \mathrm{THz}$ for a $\mathrm{QW}$ period of $55 \AA$ ( $12 \AA$ well) to $0.67 \mathrm{THz}$ for a QW period of $105 \AA$ ( $62 \AA$ well). Figure 2 plots the observed acoustic phonon angular frequency $\omega$ vs. the excited phonon wavevector $q$ which is a function of period width. A linear relation is observed. The slope of the linear relation $\omega=v q$ corresponds to the sound velocity $\mathrm{v}$ in the [0001] direction for the LA mode with a value of $6800 \mathrm{~m} / \mathrm{s}$. This value agrees well with a LA mode c-axis sound velocity of $6620 \pm 220 \mathrm{~m} / \mathrm{s}$ reported by previous measurement [5].

In our presentation, we will also present investigations on the dephasing mechanism, the phase delay of oscillation (initiation mechanism), and the spectral influence of the oscillation on the optical properties (through QCSE). Our work opens ways to the studies of the properties of acoustic phonons and their interactions with surrounding carriers in semiconductors. This huge acoustic phonon oscillation in piezoelectric field also makes it possible to generate artificially tunable strong $\mathrm{THz}$ radiation with extremely narrow linewidth in semiconductor periodic structures. This work is sponsored by National Science Council of Taiwan, R.O.C.

\section{References:}

[1] G. C. Cho, W. Kutt, and H. Kurz, Phys. Rev. Lett. 65, 764 (1990).

[2] T. K. Cheng, et al., Appl. Phys. Lett. 59, 1923 (1991).

[3] A. Yamamoto, T. Mishina, and Y. Masumoto, Phys. Rev. Lett. 73, 740 (1994).

[4] T. Dekorsy, A. Bartels, H. Kurz, and K. Köhler, in Proceeding of Ultrafast Phenomena, paper TuP27 (1998).

[5] V. A. Savastenko and A. U. Sheleg, Phys. Stat. Sol. A 48, K135 (1978).

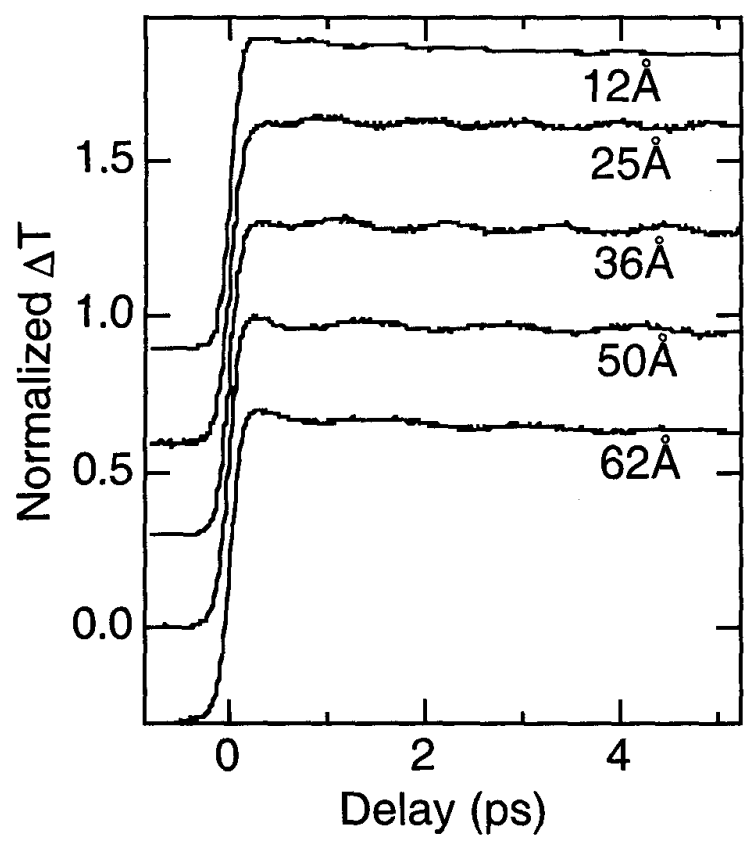

Figure 1 Measured transient transmission changes vs. probe delay. Traces are vertically displaced for clarity.

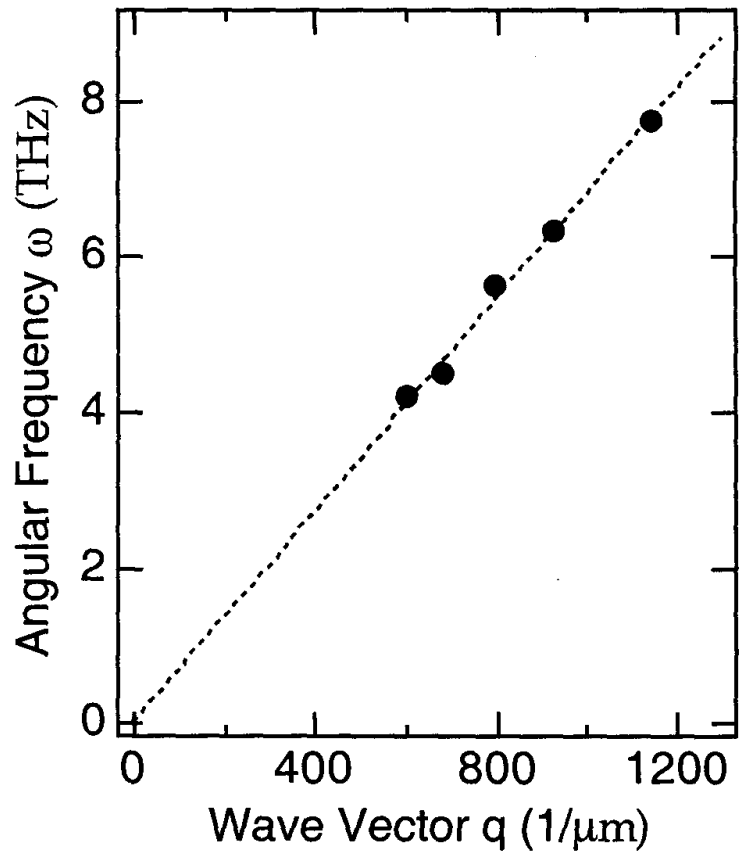

Figure 2 Measured angular frequency of oscillation vs. corresponding wavevector for different MQWs. 